\title{
Suboptimal management of rheumatoid arthritis in the Middle East and Africa: could the EULAR recommendations be the start of a solution?
}

\author{
Bassel El Zorkany • Humaid A. AlWahshi • Mohamed Hammoudeh • \\ Samar Al Emadi • Romela Benitha • Adel Al Awadhi • Elyes Bouajina • Ahmed Laatar • \\ Samir El Badawy • Marzooq Al Badi • Mustafa Al-Maini • Jamal Al Saleh • \\ Ramiz Alswailem • Mahmood Moosa Tar Mahomed Ally • Wafaa Batha • \\ Hachemi Djoudi • Ayman El Garf • Khaled El Hadidi • Mohamed El Marzouqi • \\ Musa Hadidi • Ajesh Basantharan Maharaj • Abdel Fattah Masri • Ayman Mofti • \\ Ibrahim Nahar • Clive Allan Pettipher • Catherine Elizabeth Spargo • Paul Emery
}

Received: 23 November 2012 / Accepted: 10 December 2012 /Published online: 30 December 2012

(C) Clinical Rheumatology 2012

\section{Introduction}

Rheumatoid arthritis (RA) is associated with joint destruction, functional disability and an increased risk of comorbidities and extra-articular manifestations, as well as increased mortality and reduced workforce participation [1-8]. In recent years, knowledge about the underlying pathogenesis and process of disease in RA has increased; this, coupled with significant advances in disease-modifying anti-rheumatic drugs (DMARDs) has brought about

B. El Zorkany $(\bowtie) \cdot$ S. El Badawy $\cdot$ A. El Garf $\cdot$ K. El Hadidi

Cairo University, Cairo, Egypt

e-mail: basselelzorkany@yahoo.com

H. A. AlWahshi

Royal Hospital, Muscat, Oman

M. Hammoudeh · S. Al Emadi

Hamad-Medical Corporation, Doha, Qatar

R. Benitha $\cdot$ C. A. Pettipher

Wilgeheuwel Hospital, Roodepoort, Gauteng, South Africa

\section{A. Al Awadhi}

Department of Medicine, Faculty of Medicine, Kuwait University,

Kuwait City, Kuwait

E. Bouajina

Farhat Hached Hospital, Sousse, Tunisia

A. Laatar

Mongi Slim University Hospital, Tunis El Manar University, La

Marsa, Sidi Daroud, Tunisia unprecedented opportunities for the management of the condition $[9,10]$. In order to guide rheumatologists in the use of new therapies and management strategies, the European League Against Rheumatism (EULAR) developed evidence-based recommendations for the management of RA on the principle that treatment of RA should aim for a target of remission or low disease activity as soon as possible in every patient [10]. In addition, recognising the importance of early identification of inflammatory synovitis in patients who may be at high risk of persistent and/or erosive

M. Al Badi

Security Forces Hospital, Riyadh, Saudi Arabia

M. Al-Maini

Al Mafraq Hospital, Abu Dhabi, United Arab Emirates

J. Al Saleh

Dubai Hospital, Dubai, United Arab Emirates

R. Alswailem

Riyadh Military Hospital, Riyadh, Saudi Arabia

M. M. T. M. Ally

University of Pretoria, Pretoria, South Africa

W. Batha

Red Cross Hospital, Damascus, Syria

H. Djoudi

Douera Hospital, Algiers, Algeria

M. El Marzouqi

Sheikh Khalifa Medical Center, Abu Dhabi, United Arab Emirates 
disease, and who may benefit from early effective intervention, EULAR and the American College of Rheumatology (ACR) jointly published updated classification criteria for RA with this new focus in 2010 [9]. The ACR have also recently published an update to their 2008 recommendations to reflect the refocus on early disease [11]. However, despite these great advances in understanding of the importance of early and aggressive treatment, management of RA in the Middle East and Africa remains suboptimal for a combination of reasons, and these are explored here, along with a discussion of possible solutions.

\section{Difficulties in management of RA in the Middle East and Africa}

In many countries, there exists a belief, especially at the non-specialist level, that RA is less common and milder in this region than in the developed world. Although data from this area are scant $[12,13]$, those that are available indicate that RA actually presents a significant burden in the Middle East and Africa. Indeed, the prevalence of RA in these parts of the world is reported to be in the same range as in developing countries (Table 1) [1-4, 6, 14-18]. Encouragingly, the number of patients seen by physicians with RA is increasing in some areas, such as Kenya, which may be due to improvements in health awareness among the population, a better referral system, more widespread access to rheumatologists and increasing urbanisation [19].

The group of countries represented in the region are diverse and varied, and reports on how the severity of

M. Hadidi

Shmeisani Hospital, Amman, Jordan

A. B. Maharaj

Westville Hospital, Westville, Kwazulu-Natal, South Africa

A. F. Masri

American University of Beirut, Beirut, Lebanon

A. Mofti

Al Biraa Arthritis \& Bone Clinic, Dubai, United Arab Emirates

I. Nahar

Mubarak Hospital, Jabriya, Kuwait

C. E. Spargo

Vincent Pallotti Hospital, Cape Town, South Africa

P. Emery

Leeds Institute of Molecular Medicine, University of Leeds, Leeds, UK

\section{P. Emery}

National Institute for Health Research Leeds Musculoskeletal Biomedical Research Unit, Leeds Teaching Hospitals Trust, Leeds, UK
Table 1 Reported prevalence of RA in developed and developing countries $[1-4,6,11-15]$

\begin{tabular}{lc}
\hline Country & Prevalence (\%) \\
\hline Cameroon & 0.1 \\
Saudi Arabia & 0.2 \\
Iran & $0.2-0.3$ \\
South Africa & 0.4 \\
Developed and industrialised countries & $0.3-1$ \\
\hline
\end{tabular}

disease compares to that in other regions of the world are mixed. Early studies in Arab countries reported that RA was milder in Arab populations, with fewer extra-articular manifestations [20]. A study from Congo suggested that the condition may be less severe in some African populations [21], whereas a study in Nigeria found extra-articular manifestations in just under one-third of patients [22]. In addition, research in the United Arab Emirates (UAE) found that patients presenting to a multidisciplinary musculoskeletal centre had very active disease, with mean disease activity scores (DAS) higher than those in the USA and The Netherlands [20]. Furthermore, regardless of the severity of disease, patients in the developing nations, on average, are relatively more disabled than their counterparts in industrialised nations due to the lack of social resources and state support [13].

\section{Burden of disease}

The relatively higher socioeconomic burden of diseases other than RA that are more prevalent across the Middle East and Africa can mean that the latter is perceived as less important in terms of the broader healthcare picture (Fig. 1) $[13,23]$. However, it is important to bear in mind that the prevalence and relative burden of these diseases vary considerably within the region-for example, HIV is more widespread in central and southern Africa than in North Africa and the Middle East, whereas diabetes is more widespread in the Middle East than in Africa [24]. Data on the economic burden of RA in this region are sparse, but where data do exist, such as in Morocco, RA has a considerable socioeconomic impact, with direct costs of $€ 45$ per patient per month and indirect costs per month (high rates of workplace absence, unemployment and out-of-pocket spending for medications alone) equivalent to more than the earnings from 7 days of work at the minimum wage [24]. In South Africa, functional outcomes are generally poorer in the public setting than the private setting due to lack of facilities that allow for tighter disease activity control; this results in patient overload, shorter consultations, restricted and inconsistent prescribing and erratic supply of medications in the public setting [25]. With this in mind, as well as the 
Fig. 1 Prevalence of human immunodeficiency virus $(H I V)$, cardiovascular disease, diabetes mellitus, protein-energy malnutrition, tuberculosis and rheumatoid arthritis $(R A)$ in Africa as a whole and the Eastern Mediterranean in 2004 [23]. The asterisk indicates angina and stroke survivors. Patients with protein-energy malnutrition belong to the age range of $0-4$ years

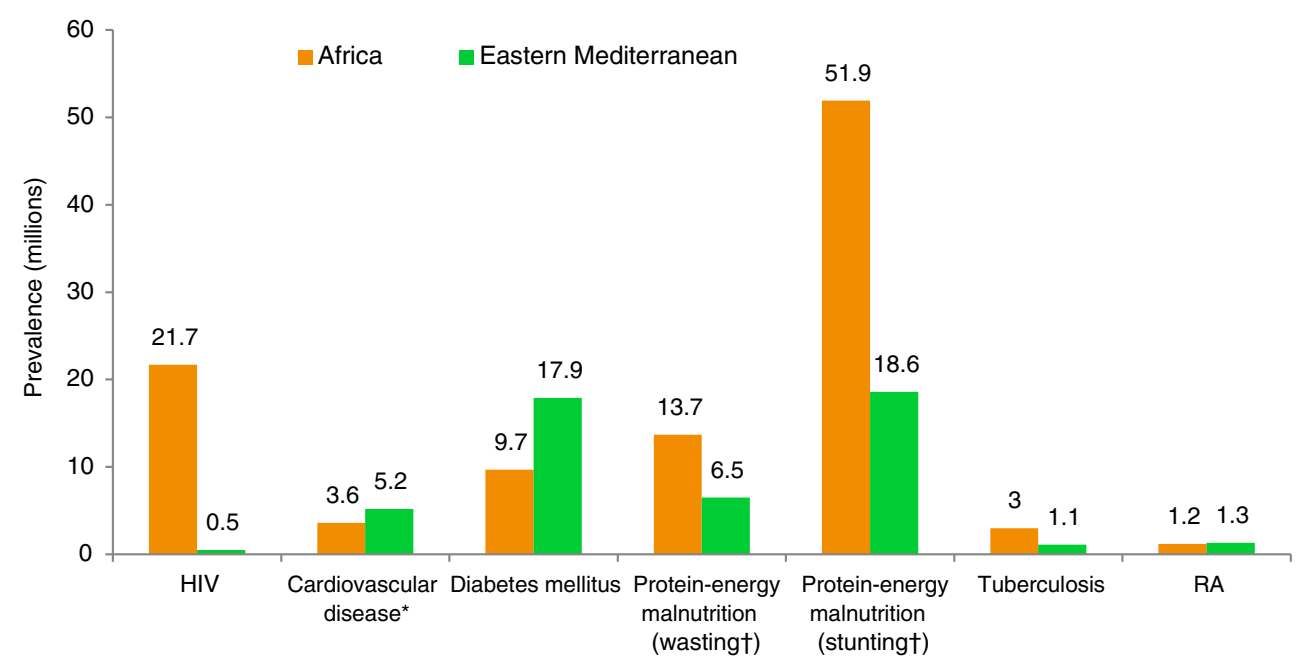

Differences in prescribing patterns for RA have been found across the Middle East and Africa. In one study from the UAE, DMARDs were being used by only $43 \%$ of patients at presentation and only $50 \%$ of patients with symptoms for $\geq 1$ year, with a lag time to use of first DMARD of 1.6 years [20]. Results were similar in Kenya, with $47 \%$ of patients receiving DMARDs - most commonly methotrexate or steroids alone or in combination-or non-steroidal antiinflammatory drugs (NSAIDs) [16]. In Morocco, although 91-98\% of patients seen at rheumatology departments were receiving DMARDs, few were receiving treatment with biologics [26]. Patients in Nigeria and South Africa generally do receive DMARDs on diagnosis, but some patients in Nigeria receive only NSAIDs over long periods, and, as mentioned previously, prescribing patterns in South Africa are not consistent [22, 25, 27].

Improved management of RA in the Middle East and Africa is therefore vital for the benefit of both patients and health economies, but something further is clearly needed in order to achieve this.

\section{Applicability of the EULAR recommendations}

Development of locally applicable and specific recommendations may represent the first step towards improving management of RA in the region. In order to do this, utilisation of existing treatment guidance, such as the 2010 EULAR recommendations, would seem to be the obvious starting point. With this in mind, 28 expert rheumatologists from 11 countries in the Middle East and North Africa (MENA) region (Algeria, Egypt, Jordan, Kuwait, Lebanon, Oman, Qatar, Saudi Arabia, Syria, Tunisia and UAE) and South Africa convened, with the main aim of determining whether the EULAR consensus recommendations are applicable and appropriate enough to be implemented in the MENA region

centres are unable to prescribe or monitor drugs [13]. 
and South Africa. The group was chaired by a former chairman and current member of the EULAR recommendations group.

The 15 EULAR recommendations take into account treatment strategies and economic implications to advocate management with a dual approach of non-pharmacological measures and pharmacological treatment involving a combination of synthetic DMARDs, steroids and biologic DMARDs (Table 2) [10]. In addition, a three-phase treatment algorithm is intended to help rheumatologists implement the recommendations (Fig. 2) [10].

The group discussed and voted on the recommendations with regard to appropriateness and local applicability for MENA and South Africa, and the main challenges facing rheumatologists in each represented country in terms of implementing the three phases of the EULAR treatment algorithm were reviewed. Finally, opportunities and obstacles to implementation of the recommendations were considered.

In order to successfully utilise the EULAR treatment recommendations as the first step in development, it is important to understand whether healthcare physicians in these regions are already aware of the recommendations and if they are attempting to implement them. In this respect, the first signs were favourable, as the members of the group are very supportive of the EULAR recommendations. However, this group has a specific interest in RA, and it was thought that general awareness among the wider population of rheumatologists and other clinicians and stakeholders without a special interest in RA was low. Although both agreement with and implementation of some of the 15 treatment recommendations was believed to be high, other recommendations were felt not to be as widely implemented, even when agreement remained high (Fig. 3). Some common themes, as well as some interesting differences, emerged during these discussions, particularly relating to the recommendations that were felt to be most difficult to implement. Significant differences across the region in terms of economics, cultural issues, educational issues, healthcare access and health priorities were noted, in agreement with the sparse data discussed previously.

Table 2 EULAR recommendations

European League Against Rheumatism (EULAR) treatment recommendations [10]

1. Treatment with synthetic DMARDs should be started as soon as the diagnosis of RA is made

2. Treatment should be aimed at reaching a target of remission or low disease activity as soon as possible in every patient; as long as the target has not been reached, treatment should be adjusted by frequent (every 1-3 months) and strict monitoring

3. Methotrexate should be part of the first treatment strategy in patients with active RA

4. When methotrexate contraindications (or intolerance) are present, the following DMARDs should be considered as part of the (first) treatment strategy: leflunomide, sulfasalazine or injectable gold

5. In DMARD-naïve patients, irrespective of the addition of glucocorticoids, synthetic DMARD monotherapy rather than combination therapy of synthetic DMARDs may be applied

6. Glucocorticoids added at low to moderately high doses to synthetic DMARD monotherapy (or combinations of synthetic DMARDs) provide benefit as initial short-term treatment but should be tapered as rapidly as clinically feasible

7. If the treatment target is not achieved with the first DMARD strategy, addition of a biologic DMARD should be considered when poor prognostic factors are present; in the absence of poor prognostic factors, switching to another synthetic DMARD strategy should be considered

8. In patients responding insufficiently to methotrexate and/or other synthetic DMARDs with or without glucocorticoids, biologic DMARDs should be started; current practise would be to start a TNF inhibitor (adalimumab, certolizumab, etanercept, golimumab, infliximab) which should be combined with methotrexate

9. Patients with RA for whom a first TNF inhibitor has failed should receive another TNF inhibitor: abatacept, rituximab or tocilizumab

10. In cases of refractory severe RA or contraindications to biologic agents or the previously mentioned synthetic DMARDs, the following synthetic DMARDs might be also considered, as monotherapy or in combination with some of the above: azathioprine or ciclosporin A (or, exceptionally, cyclophosphamide)

11. Intensive medication strategies should be considered in every patient, although patients with poor prognostic factors have more to gain

12. If a patient is in persistent remission, after having tapered glucocorticoids, one can consider tapering biologic DMARDs, especially if this treatment is combined with a synthetic DMARD

13. In cases of sustained long-term remission, cautious titration of synthetic DMARD dose could be considered, as a shared decision between patient and doctor

14. DMARD-naïve patients with poor prognostic markers might be considered for combination therapy of methotrexate plus a biologic agent

15. When adjusting treatment, factors apart from disease activity, such as progression of structural damage, comorbidities and safety concerns, should be taken into account

$D M A R D s$ disease-modifying anti-rheumatic drugs, $R A$ rheumatoid arthritis, TNF tumour necrosis factor 


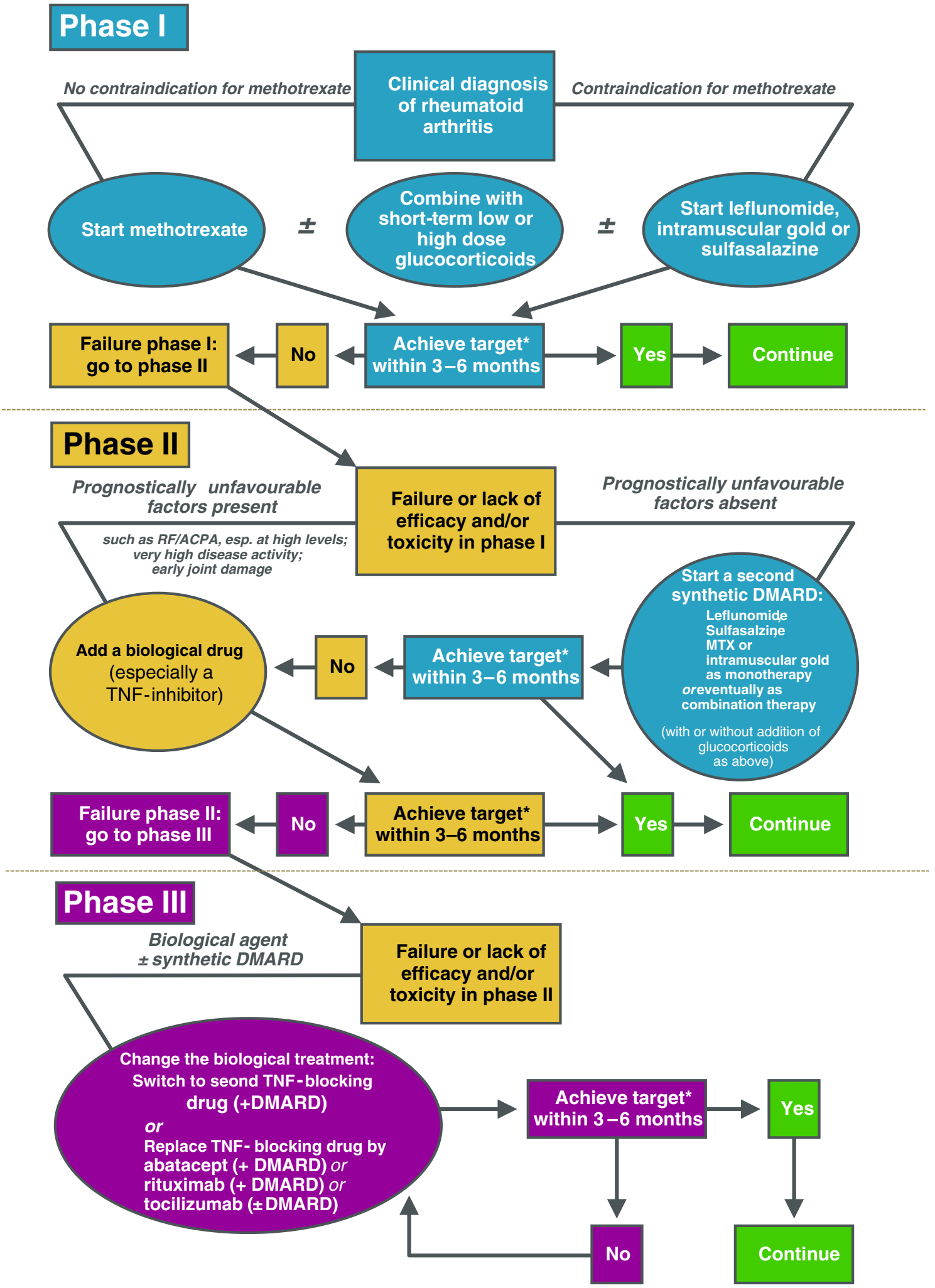

*The treatment target is clinical remission or, if remission is unlikely to be achievable, at least low disease activity

Fig. 2 Algorithm based on European League Against Rheumatism recommendations for the management of rheumatoid arthritis. Reproduced with permission from Smolen et al. [10] 
Fig. 3 Results from voting by members of the group on the European League Against Rheumatism recommendations. Votes were classified as 'agree' if the response was 'agree' or 'strongly agree' and 'disagree' if the response was 'disagree' or 'strongly disagree', and a response of 'neutral' was recorded as such. Votes were classified as 'implemented' if the response was 'yes, by all rheumatologists' or 'yes, by most rheumatologists' and as 'not implemented' if the response was 'yes, but by few rheumatologists' or 'no'

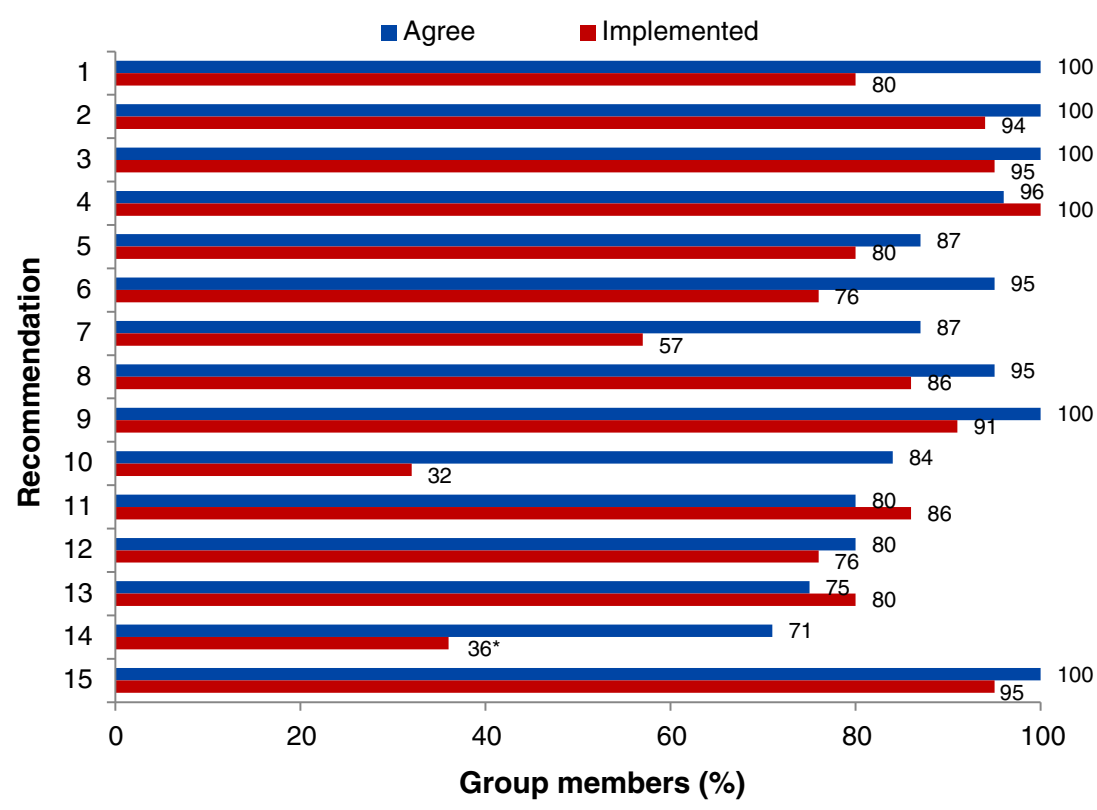

Recommendations 7 and 14 which relate to the introduction of a biologic in patients who fail to reach targets with DMARD therapy, or who have poor prognostic markerswere identified as being difficult to implement. Despite $71 \%$ agreement with recommendation 14 (DMARD-naïve patients with poor prognostic markers might be considered for combination therapy of methotrexate plus a biologic agent), implementation of this recommendation was perceived to be very low (36\%), with the main obstacle being access to the drugs. In South Africa, this is a problem both in state hospitals, where access is mostly through clinical trials, and in private healthcare, where access is limited due to funders' rules on the use of biologics. Across the region, the low use of biologics may be in part due to the costs of these drugs and the monitoring required with their use, as well as concerns about the risks of reactivation of tuberculosis in populations where it is endemic [13].

Other recommendations that were felt to be difficult to implement in clinical practise in the Middle East and Africa included 1, 6 and 10. These recommendations cover a variety of themes but all advocate aggressive management that requires early use of intensive therapies and tight disease control through frequent monitoring and consideration of factors other than disease activity. The reasons for the difficulty in implementing these are multiple. In Egypt, the lack of time to perform DAS and health assessment questionnaire measurements, lack of assistants, lack of awareness, misinformation (for example, about drug toxicity and comorbidities), economic issues and the local practise of exhausting synthetic DMARDs and glucocorticoids before a biologic is introduced were the key issues. In South Africa, delayed referral, shortages of rheumatologists and rheumatology nurses and the need to prioritise funding to diseases with other higher burdens, such as tuberculosis, HIV and hepatitis B, were major obstacles. In the UAE, the need for earlier access to patients is a problem due to the diverse nature of the population and the numerous healthcare systems in place. Lack of local data in Kuwait and Oman is a major barrier to implementing some of the recommendations. Members from countries with high proportions of expatriates felt that issues around lack of appropriate insurance need to be addressed to encourage appropriate treatment in line with the recommendations.

According to 15 of the experts present, the main challenge to implementation of Phase I of the treatment algorithm (Fig. 1) [10] is that patients are not referred early enough from general practise. Other challenges, in descending order, included lack of early RA clinics, lack of standardised disease assessment measurement in clinical practise, lack of specialist rheumatology nurses/care teams and lack of knowledge of EULAR diagnostic criteria. The main challenge to implementation of Phases II and III of the treatment algorithm was lack of access to biologic therapies, according to 13 and 14 expert members, respectively. Other reported challenges to Phase II were lack of specialist rheumatology nurses/care team, lack of local guidelines regarding initiation of biologic therapies, lack of standardised disease assessment measurement in clinical practise and patients/physicians being wary of the risks versus benefits of biologic therapy. Other challenges to Phase III included lack of local guidelines regarding initiation of biologic therapies and patients not attending follow-up appointments.

\section{Next steps in improving management in the Middle East and Africa}

Issues specific to this region, and in some cases specific to individual countries, create barriers to the widespread 
implementation of the EULAR treatment recommendations in clinical practise, with key obstacles to their implementation including lack of timely referral to rheumatologists, suboptimal use of synthetic DMARDs and poor access to biologics. Many of the issues identified here were highlighted previously in 2003, in Kalla and Tikly's review of RA across the wider developing world [13]. Little seems to have changed in the Middle East and Africa over the 9 years since that preliminary review was published, so efforts to optimise the management of RA in this region need to be re-energised and addressed through a multi-pronged approach.

\section{Epidemiological data collection}

Misunderstanding of, misconceptions about and lack of awareness around the prevalence, severity and burden of RA in this region seem to be at the root of some of the barriers to its optimal management. The lack of data on the prevalence, severity, impact and burden of RA in the region leaves questions unanswered, which provides physicians with sufficient doubt to focus their efforts on other diseases that may seem to be a higher priority due to solid local evidence. It is therefore essential for epidemiological data on RA to be collected from every country in the Middle East and Africa to increase understanding of the intra- and inter-regional variability of the disease [12] and highlight the true impact for patients, healthcare systems and economies. A collaborative approach to collecting this data could prove to be the most fruitful, enabling pooling of resources and centralisation of administration, which would allow countries with limited resources to participate.

\section{Education initiatives}

A variety of direct and indirect educational initiatives by local rheumatological societies would be crucial in raising awareness of the disease and addressing misunderstanding and misconceptions. Education programmes for healthcare professionals (including GPs, registrars and nurses) would increase awareness of the prevalence of RA and knowledge of the signs of early inflammatory synovitis, which could lead to increased suspicion of the disease and earlier diagnosis and referral. Education about the burden and impact of RA on patients and society as a whole, particularly as the disease progresses, could drive earlier referral and more aggressive management. In addition, with many patients unaware of RA, an education programme to increase public awareness of the disease, and the rheumatology speciality as a whole, could encourage patients to present to their primary-care provider to discuss their symptoms, treatment options and the possibility of specialist referral.

\section{Development of regional guidelines}

As has been highlighted already, the EULAR recommendations are, in general, relevant and applicable to many countries in this region. In this regard, initiatives to increase awareness and assist in implementation of these recommendations locally should be undertaken by local and regional rheumatology societies. In addition, as it has been shown that challenges hampering the implementation of these recommendations exist in some areas. Again, local and regional societies and bodies should collaborate to identify specific needs in their region and begin development of local guidelines, as locally applicable guidelines and recommendations could facilitate an increase in implementation of an evidence-based approach, which could mean improved patient outcomes.

\section{Access to therapy}

Facilitation of treatment access in line with the EULAR recommendations is a key to optimal management of RA. Early initiation of DMARDs with methotrexate used as an anchor DMARD and optimisation of methotrexate use, perhaps through subcutaneous dosing, could be useful strategies moving forward; this would encourage implementation of recommendations early in the treatment pathway, giving a base upon which later treatments and recommendations could be built. The EULAR recommendations are already accepted by many rheumatological societies in the region, but implementation is not high due to the perceived cost of treatment in line with these recommendations, so education of payers and other stakeholders will be important to increase awareness of the indirect benefits of optimal treatment, including its cost benefit and cost effectiveness. The development and use of 'burden of disease' and pharmacoeconomic models could help to demonstrate the benefits of investing in aggressive treatments earlier in the disease process to reduce costs and burden later in the patient's journey with RA. In addition, implementing regulation to disallow treatment unless patients are evaluated by rheumatologists would encourage adherence with the recommendations; however, as highlighted earlier, lack of time, excessive workload and lack of appropriate staffing are recurring themes, so there is also an urgent need to create more rheumatology training posts, establish more posts for trained assistants and nursing staff and create a strong training programme for nurses so that more healthcare 
professionals are able to identify and take on the dayto-day treatment and monitoring of patients with RA.

\section{Summary}

Although the prevalence of RA in the Middle East and Africa is comparable with that in other parts of the world, evidence indicates that its management in this region is suboptimal for a variety of reasons, including misconceptions and misunderstandings about the disease's prevalence and severity in the region, compounded by the lack of local epidemiological and health-economic data around the disease; the perception that RA is a low priority compared with other more prevalent conditions; delayed diagnosis, referral and treatment; and a lack of a region-specific, evidencebased management approach. In the absence of such an approach, the EULAR treatment recommendations may provide a useful starting point for the creation of guidelines to suit local circumstances. However, although agreement with the EULAR recommendations is high, many barriers prevent their implementation in clinical practise, including lack of timely referral to rheumatologists; suboptimal use of synthetic DMARDs; poor access to biologics; lack of awareness of the burden of RA among healthcare professionals, patients and payers; and lack of appropriate staffing levels.

To optimise the management of RA in the Middle East and Africa, will require a multi-pronged approach from a diverse group of stakeholders - including local, national and regional societies, such as the African League of Associations in Rheumatology and International League of Associations for Rheumatology, and service providers - to collect data on the epidemiology and burden of the disease; to increase awareness of RA and its burden among healthcare professionals, payers and patients through various educational programmes; to encourage early referral and optimise use of DMARDs by promoting the EULAR treatment recommendations; to encourage the development of locally applicable guidelines based on the EULAR treatment recommendations; and to facilitate access to drugs and the healthcare professionals who can prescribe and monitor them.

Acknowledgments Medical writing support was provided by Jemma Lough at Synergy and was funded by Pfizer. This paper was based on proceedings of an advisory board which was funded by Pfizer.

Disclosures Ramiz Alswailem received consulting honoraria from Pfizer, Abbott, Bristol-Myers Squibb and MSD. Bassel El Zorkany received speaker's and advisory honoraria from Abbott, Aventis, BMS, Lilly, MSD, Novartis, Pfizer, Roche and Servier. Mohammed Hammoudeh has received research support from Pfizer, Wyeth, Roche and Schering. Paul Emery has given expert advice and received honoraria from Merck, Pfizer, Abbott, UCB, Roche, Novartis and BMS.

\section{References}

1. Lee DM, Weinblatt ME (2001) Rheumatoid arthritis. Lancet 358:903-911

2. Scott DL, Wolfe F, Huizinga TWJ (2010) Rheumatoid arthritis. Lancet 376:1094-1108

3. World Health Organization (2011) Chronic diseases and health promotion: chronic rheumatic conditions. WHO, Geneva

4. Woolf AD, Pfleger B (2003) Burden of musculoskeletal conditions. Bull World Heath Org 81:646-656

5. Callahan LF, Pincus T (1995) Mortality in the rheumatic diseases. Arthritis Care Res 8:229-241

6. Gabriel SE (2001) The epidemiology of rheumatoid arthritis. Rheum Dis Clin North Am 27:269-281

7. Minaur NJ, Jacoby RK, Cosh JA, Taylor G, Rasker JJ (2004) Outcome after 40 years with rheumatoid arthritis: a prospective study of function, disease activity, and mortality. J Rheumatol 31 (Suppl 69):3-8

8. Lundkvist J, Kastäng F, Kobelt G (2008) The burden of rheumatoid arthritis and access to treatment: health burden and costs. Eur J Health Econ 8(Suppl 2):S49-S60

9. Aletaha D, Neogi T, Silman AJ, Funovits J, Felson DT, Bingham CO, Birnbaum NS, Burmester GR, Bykerk BP, Cohen MD, Combe B, Costenbader KH, Dougados M, Emery P, Ferraccioli G, Hazes JMW, Hobbs K, Huizinga TWJ, Kavanaugh A, Kay J, Kvien TK, Laing T, Mease P, Ménard HA, Moreland LW, Naden RL, Pincus T, Smolen JS, Stanislawska-Biernat E, Symmons D, Tak PP, Upchurch KS, Vencovský J, Wolfe F, Hawker G (2010) 2010 Rheumatoid arthritis classification criteria: an American College of Rheumatology/European League Against Rheumatism collaborative initiative. Ann Rheum Dis 69:1580-1588

10. Smolen JS, Landewé R, Breedveld FC, Dougados M, Emery P, Gaujoux-Viala C, Gorter S, Knevel R, Nam J, Schoels M, Aletaha D, Buch M, Gossec L, Huizinga T, Bijlsma JW, Burmester G, Combe B, Cutolo M, Gabay C, Gomez-Reino R, Kouloumas M, Kvien TK, Martin-Mola E, McInnes I, Pavelka K, van Riel P, Scholte M, Scott DL, Sokka T, Valesini G, van Vollenhoven R, Winthrop KL, Wong J, Zink A, van der Heijde D (2010) EULAR recommendations for the management of rheumatoid arthritis with synthetic and biological disease-modifying antirheumatic drugs. Ann Rheum Dis 69:964-975

11. Singh JA, Furst DE, Bharat A, Curtis JR, Kavanaugh AF, Kremer JM, Moreland LW, O'Dell J, Winthrop KL, Beukelman T, Bridges SL Jr, Chatham WW, Paulus HE, Suarez-Almazor M, Bombardier C, Dougados M, Khanna D, King CM, Leong AL, Matteson EL, Schousboe JT, Moynihan E, Kolba KS, Jain A, Volkmann ER, Agrawal H, Bae S, Mudano AS, Patkar NM, Saag KG (2012) 2012 update of the 2008 American College of Rheumatology recommendations for the use of disease-modifying antirheumatic drugs and biologic agents in the treatment of rheumatoid arthritis. Arth Care Res 64:625-639

12. World Health Organization (2003) The burden of musculoskeletal conditions at the start of the new millennium. WHO technical report series 919. Geneva: WHO

13. Kalla AA, Tikly M (2003) Rheumatoid arthritis in the developing world. Best Pract Res Clin Rheumatol 17:863-875

14. Singwe-Ngandeu M, Meli J, Ntsiba H, Nouédoui C, Yollo AV, Sida MB, Muna WF (2007) Rheumatic diseases in patients attending a clinic at a referral hospital in Yaounde, Cameroon. East Afr Med J 84:404-409

15. Al-Dalaan A, Al Ballaa S, Bahabri S, Biyari T, Sukait MA, Mousa M (1998) The prevalence of rheumatoid arthritis in the Qassim region of Saudi Arabia. Ann Saudi Med 18:396-397

16. Davatchi F, Jamshidi AR, Banihashemi AT, Gholami J, Forouzanfar MH, Akhlaghi M, Barghamdi M, Noorolahzadeh E, Khabazi AR, 
Salesi M, Salari AH, Karimifar M, Essalat-Manesh K, Hajialiloo M, Soroosh M, Farzad F, Moussavi HR, Samadi F, Ghaznavi K, Asgharifard H, Zangiabadi AH, Shahram F, Nadji A, Akbarian M, Gharibdoost F (2008) WHO-ILAR COPCORD Study (Stage 1, Urban Study) in Iran. J Rheumatol 35:1384-1390

17. Davatchi F, Banihashemi AT, Gholami J, Faezi ST, Forouzanfar MH, Salesi M, Karimifar M, Essalatmanesh K, Barghamdi M, Noorolahzadeh E, Dahaghin S, Rasker JJ (2009) The prevalence of musculoskeletal complaints in a rural area in Iran: a WHOILAR COPCORD study (stage 1, rural area) in Iran. Clin Rheumatol 28:1267-1274

18. Parker R, Jelsma J (2010) The prevalence and functional impact of musculoskeletal conditions amongst clients of a primary health care facility in an under-resourced area of Cape Town. BMC Musculoskelet Disord 11:2

19. Owino BO, Oyoo GO, Otieno CF (2009) Socio-demographic and clinical aspects of rheumatoid arthritis. East Afr Med J 86:204-211

20. Badsha H, Kong KO, Tak PP (2008) Rheumatoid arthritis in the United Arab Emirates. Clin Rheumatol 27:739-742
21. Malemba JJ, Mbuyi-Muamba JM (2008) Clinical and epidemiological features of rheumatic diseases in patients attending the university hospital in Kinshasa. Clin Rheumatol 27:47-54

22. Adelowo OO, Ojo O, Oduenyi O, Okwara CC (2010) Rheumatoid arthritis among Nigerians: the first 200 patients from a rheumatology clinic. Clin Rheumatol 20:593-597

23. World Health Organization (2008) The global burden of disease: 2004 update. WHO, Geneva

24. World Health Organization (2011). Global health observatory data repository: number of people (all ages) living with HIV. Geneva: WHO. Available at: http://apps.who.int/ghodata/?vid=22100\#

25. Solomon A, Christian BF, Dessein PH, Stanwix AE (2005) The need for tighter rheumatoid arthritis control in a South African Public Health Care Center. Semin Arthritis Rheum 35:122-131

26. Rkain H, Allali F, Jroundi I, Hajjaj-Hassouni N (2006) Socioeconomic impact of rheumatoid arthritis in Morocco. Joint Bone Spine $73: 278-283$

27. Tikly M, Zannettou N, Hopley M (2003) A longitudinal study of rheumatoid arthritis in South Africans. Med Gen Med 5(1):2 